\title{
COMPLETENESS OF THE BETHE ANSATZ FOR THE PERIODIC ISOTROPIC HEISENBERG MODEL
}

\author{
V. TARASOV
}

\begin{abstract}
For the periodic isotropic Heisenberg model with arbitrary spins and inhomogeneities, we describe the system of algebraic equations whose solutions are in bijection with eigenvalues of the transfer-matrix. The system describes pairs of polynomials with the given discrete Wronskian (Casorati determinant) and additional divisibility conditions on discrete Wronskians with multiple steps. If the polynomial of the smaller degree in the pair is coprime with the Wronskian, this system turns into the standard Bethe ansatz equations. Moreover, if the transfer-matrix is diagonalizable, then its spectrum is necessarily simple modulo natural degeneration.
\end{abstract}

\section{To the memory of Ludwig Dmitrievich Faddeev}

\section{INTRODUCTION}

The problem to find eigenvalues and eigenvectors of the Hamiltonian of the Heisenberg model was first addressed by $\mathrm{H}$. Bethe [Be] who looked for the eigenvectors in a certain specific form. His method and its various further extensions are nowadays called the Bethe ansatz. The current literature on the Heisenberg model and its counterparts in statistical mechanics, the six- and eight-vertex models, is enormous, so we limit our references here just to two classical books [B1], [KBI].

The Hamiltonian of the Heisenberg model can be included into a one-parameter family of commuting linear operators called the transfer-matrix, see [B1], [FT], $[\mathrm{KBI}]$, and the actual problem is to find eigenvalues and eigenvectors of the transfer-matrix. This can be done by the algebraic Bethe ansatz, see $[\mathrm{FT}],[\mathrm{FT}]$, in the framework of the quantum inverse scattering method (QISM). Moreover, the QISM produces an integrable higher spin generalization of the Heisenberg model $[\mathrm{KRS}],[\mathrm{KR}]$, that can be solved by the algebraic Bethe ansatz as well.

The Bethe ansatz approach reduces the problem of diagonalizing the transfer-matrix of the Heisenberg model to solving a system of algebraic equations, called the Bethe ansatz equations, and gives a recipe to construct an eigenvector and an eigenvalue of the transfermatrix given a solution of the Bethe ansatz equations. In fact, not all solutions of the Bethe ansatz equations equations are equally good, and there are also selection rules on how to pick up acceptable solutions, that is, those that are better than others. It is desirable that all eigenvectors of the transfer-matrix can be obtained from acceptable solutions of the Bethe ansatz equations, and whether it is possible to achieve, say, by tuning the selection rules, is the question of completeness of the Bethe ansatz for the Heisenberg model on a finite lattice.

The completeness question was discussed in [Be] by $\mathrm{H}$. Bethe himself and many times since then by various authors, see for instance, [Ki], [LS], [TV], [B2] and references therein. However, the question is not considered to be closed yet with more papers dealing with different aspects of the problem, see for example [DG], [GN], [KS].

This is the author's manuscript of the article published in final edited form as:

Tarasov, V. (2018). Completeness of the Bethe Ansatz for the Periodic Isotropic Heisenberg Model. Reviews in Mathematical Physics, 30(08), 1840018. http://dx.doi.org/10.1142/S0129055X18400184 
In this paper we revisit the question of completeness of the Bethe ansatz and present the results that were obtained in the author's previous works with E. Mukhin and A.Varchenko, together with recent progress for the higher spin model. For the already established statements, the goal is to translate them from the language of commutative algebra to a more familiar and accessible language of linear algebra, making the results easier to appreciate. To this end, the assertions might be slightly weakened for the sake of simplicity of presentation. Also, because of the change of language, the statements need not look the same as in the original papers referred to, or even it might be no straightforward counterparts there at all. To keep the paper shorter, details of translation are mostly omitted and the proofs of new results will appear elsewhere.

It turns out that the key to achieving the goal of completeness of the Bethe ansatz for the Heisenberg model is an appropriate modification of the standard Bethe ansatz equations For a periodic spin-1/2 chain of length $n$ with inhomogeneities $z_{1}, \ldots, z_{n}$, the right system of algebraic equations describes the problem of finding a pair of polynomials $p(u), q(u)$ in one variable with the prescribed discrete Wronskian (Casorati determinant),

$$
p\left(u+\frac{i}{2}\right) q\left(u-\frac{i}{2}\right)-p\left(u-\frac{i}{2}\right) q\left(u+\frac{i}{2}\right)=\prod_{s=1}^{n}\left(u-z_{s}\right),
$$

see [MTV5], [MTV9]. For a periodic model, the transfer-matrix is SL(2)-invariant, that determines a natural degeneration of its spectrum. And modulo this degeneration, eigenvectors and eigenvalues of the transfer-matrix are in bijection with classes of pairs of polynomials $p, q$ satisfying (1.1), provided the pairs $p_{1}, q_{1}$ and $p_{2}, q_{2}$ are in the same class if they span the same two-dimensional vector space. In particular, the spectrum of the transfer-matrix is simple modulo the natural degeneration induced by the SL(2)-invariance of the model.

Similar result holds for the periodic higher spin chains. In this case, the spectrum of the transfer-matrix is also simple modulo the natural degeneration, and eigenvalues of the transfer-matrix are in bijection with the classes of pairs of polynomials $p(u), q(u)$ that have the prescribed discrete Wronskian $p\left(u+\frac{i}{2}\right) q\left(u-\frac{i}{2}\right)-p\left(u-\frac{i}{2}\right) q\left(u+\frac{i}{2}\right)$ and whose discrete Wronskians with multiple steps

$$
p\left(u+m \frac{i}{2}\right) q\left(u-m \frac{i}{2}\right)-p\left(u-m \frac{i}{2}\right) q\left(u+m \frac{i}{2}\right)
$$

are divisible by certain given polynomials. For the spin chain with the largest spin $\ell$, the number $m$ in (1.2) runs through integers between 1 and $2 \ell$.

This result has a grain of surprise. One might expect that additional conditions on polynomials $p(u), q(u)$ for higher spin models could be formulated in terms of the Baxter equation

$$
a(u) p(u-i)+d(u) p(u+i)=E(u) p(u) .
$$

However, what one can get this way is only the divisibility condition for the discrete Wronskian (1.2) with $m=2$, and it is not sufficient for most of spin chains that contain spins higher than 1, see an example in Section 6.

The Bethe ansatz for the Gaudin model is understood nowadays much better than for lattice integrable models. The completeness of the Bethe ansatz is proved for the Gaudin model associated to any semisimple Lie algebra and any tensor product of its finite-dimensional representations, see [MTV0], $[\mathrm{R}]$. The proof in [MTV0] relies upon the reformulation of the Bethe ansatz equations as the intersection equations for Schubert cells in the Grassmannians 
relative to osculating flags. For lattice integrable models, the Schubert calculus approach is applicable only partially and does not cover the most interesting cases, in particular, the homogeneous model. The divisibility conditions for discrete Wronskians with multiple steps do describe the intersection of Schubert cells in Grassmannians relative to secant flags once this picture is applicable. And it is an interesting question to find an appropriate geometric picture for the divisibility conditions that works for all cases.

In this note we consider the most rigid and degenerate case of the isotropic Heisenberg model (XXX model) with periodic boundary conditions. The results extend almost literally with due modifications to the case of the the $X X X$ model with quasiperiodic (a.k.a. twisted) boundary conditions. An interesting question is to work out the case of the $X X X$ model with open boundary conditions solvable by the Bethe ansatz. It turns out that solving the Bethe ansatz equations for a model with open boundary conditions is equivalent to imposing an additional symmetry on solutions of the Bethe ansatz equations for the "doubled" model with (quasi) periodic boundary conditions.

The case of the $X X Z$ model, which is mostly discussed in the literature for completeness of the Bethe ansatz, is studied less than the case of the $X X X$ model in the direction described in the paper. For generic anisotropy, the case of the $X X Z$ model is likely to have only technical subtleties rather than principal difficulties compared to the $X X X$ case. For generic quasiperiodic boundary conditions, including the pure periodic case, the results are expected to be similar to the case of the quasiperiodic $X X X$ model, while for certain specific quasiperiodic boundary conditions, degeneration of the spectrum of the transfer-matrix can occur resembling the case of the periodic $X X X$ model. On the other hand, the case of the $X X Z$ model at roots of unity is more sophisticated due to the known large degeneracy of eigenvalues of the transfer-matrix. Nevertheless, it is plausible for this case too that the appropriate form of the Bethe ansatz equations yielding completeness of the Bethe ansatz describes pairs of functions with the given discrete Wronskian.

The plan of the paper is as follows. Section 2 describes the higher spin $X X X$ model. In Section 3 we review the algebraic Bethe ansatz method and start to discuss the completeness question. In Section 4 we remind reformulation of the Bethe ansatz equations via discrete Wronskians of pairs of polynomials and the second order difference equations with a basis of polynomial solutions. The results on completeness of the Bethe ansatz for spin-1/2 models are given in Section 5, and for higher spin models in Section 6.

The author was supported in part by the Simons Foundation grant \#430235.

\section{HigheR SPIN XXX MODEL}

Let $S^{+}, S^{-}, S^{3}$ be the generators of the Lie algebra $\mathfrak{s l}_{2}$ :

$$
\left[S^{3}, S^{ \pm}\right]= \pm S^{ \pm}, \quad\left[S^{+}, S^{-}\right]=2 S^{3} .
$$

Let $V_{\ell}$ be the irreducible $(2 \ell+1)$-dimensional representation of $\mathfrak{s l}_{2}$, that is, the representation of spin $\ell$. Denote by $w_{\ell}$ the highest weight vector of $V_{\ell}: S^{+} w_{\ell}=0, S^{3} w_{\ell}=\ell w_{\ell}$.

The space of states of the higher spin $X X X$ model with spins $\ell_{1}, \ldots, \ell_{n}$ is the tensor product $\mathcal{H}=V_{\ell_{1}} \otimes \ldots \otimes V_{\ell_{n}}$. The local $L$-operator of the model is

$$
L_{a}(u)=\left(\begin{array}{cc}
u+i S_{a}^{3} & i S_{a}^{-} \\
i S_{a}^{+} & u-i S_{a}^{3}
\end{array}\right) \in \operatorname{End}\left(\mathbb{C}^{2} \otimes \mathcal{H}\right),
$$


where $S_{a}^{ \pm}, S_{a}^{3}$ are linear operators acting as $S^{ \pm}, S^{3}$, respectively, on the $a$-th tensor factor and as the identity operator on the other tensor factors.

The monodromy matrix of the $X X X$ model with inhomogeneities $z_{1}, \ldots, z_{n}$ equals

$$
T(u)=L_{n}\left(u-z_{n}\right) \ldots L_{1}\left(u-z_{1}\right)=\left(\begin{array}{cc}
A(u) & B(u) \\
C(u) & D(u)
\end{array}\right),
$$

and for the model with periodic boundary conditions, the transfer-matrix is

$$
\mathcal{T}(u)=\operatorname{tr} T(u)=A(u)+D(u) .
$$

It is known that the transfer-matrix forms a commutative family of operators on $\mathcal{H}$ :

$$
\mathcal{T}(u) \mathcal{T}(v)=\mathcal{T}(v) \mathcal{T}(u)
$$

for any $u, v$. The question we are interested in is to find eigenvectors and eigenvalues of the transfer-matrix.

The Lie algebra $\mathfrak{s l}_{2}$ acts naturally on the space $\mathcal{H}$, the generator $S^{\nu}$ acting as the sum $S_{1}^{\nu}+\ldots+S_{n}^{\nu}$. The transfer-matrix $\mathcal{T}(u)$ commutes with this action,

$$
\left[\mathcal{T}(u), S^{\nu}\right]=0, \quad \nu=+,-, 3,
$$

hence, every eigenspace of $\mathcal{T}(u)$ is invariant under the action of $\mathfrak{s l}_{2}$. To eliminate this degeneracy of the spectrum of the transfer-matrix, we consider the action of $\mathcal{T}(u)$ on the multiplicity spaces of irreducible representations of $\mathfrak{s l}_{2}$ in the representation $\mathcal{H}$. The multiplicity spaces are conveniently visualized as the subspaces of weight singular vectors

$$
\mathcal{H}_{k}=\left\{v \in \mathcal{H} \mid S^{+} v=0, S^{3} v=\left(\ell_{1}+\ldots+\ell_{n}-k\right) v\right\}
$$

that are invariant under the action of $\mathcal{T}(u)$ due to $(2.2)$. The subspace $\mathcal{H}_{k}$ is not trivial if only if $k$ is an integer such that $0 \leqslant k \leqslant \ell_{1}+\ldots+\ell_{n}$.

We say that the spectrum of $\mathcal{T}(u)$ is simple modulo natural degeneration if $\mathcal{T}(u)$ is diagonalizable and all its eigenspaces are irreducible representations of $\mathfrak{s l}_{2}$.

We will assume that the inhomogeneities $z_{1}, \ldots, z_{n}$ satisfy the following condition:

Let $X_{s}=\left\{z_{s}-i \ell_{s}, z_{s}-i\left(\ell_{s}-1\right), \ldots, z_{s}+i \ell_{s}\right\}, s=1, \ldots, n$. Then for any $r, s=1$, $\ldots, n$, the sets $X_{r}$ and $X_{s}$ either do not intersect or one of them is a subset of the other.

This condition means that the space $\mathcal{H}$ has no invariant subspaces under the action of the entries $A(u), B(u), C(u), D(u)$ of $T(u)$, see [T]. In other words, $T(u)$ defines an irreducible representation of the Yangian $Y\left(\mathfrak{g l}_{2}\right)$ on $\mathcal{H}$. It is known that under condition (2.3), the equivalence class of this representation does not depend on the order of pairs $\left(\ell_{1}, z_{1}\right), \ldots$, $\left(\ell_{n}, z_{n}\right)$. In particular, the spectrum of $\mathcal{T}(u)$ remains unchanged.

We will not assume that $z_{1}, \ldots, z_{n}$ satisfy some kind of reality conditions. So the transfermatrix $\mathcal{T}(u)$ might be not diagonalizable. The results on completeness of the Bethe ansatz extends to such cases as well, but for the sake of simplicity, they are not completely covered in this paper.

It is relatively easy to show that $\mathcal{T}(u)$ is diagonalizable if all $z_{1}, \ldots, z_{n}$ are sufficiently large and after a permutation satisfy inequalities $\left|z_{1}-z_{2}\right| \ll\left|z_{2}-z_{3}\right| \ll \ldots \ll\left|z_{n-1}-z_{n}\right|$. 
Thus $\mathcal{T}(u)$ is diagonalizable for generic $z_{1}, \ldots, z_{n}$. The description of the exceptional locus of $z_{1}, \ldots, z_{n}$ is a very interesting problem, but it is out of reach for the moment. However, there are two cases when $\mathcal{T}(u)$ is known to be diagonalizable because its coefficients are normal operators relative to some positive definite scalar product on $\mathcal{H}$, see [MTV10]. The cases are as follows:

(2.4) There exists a number $c$ such that the polynomial $W(u-c)$ has real coefficients and $\left|\operatorname{Im}\left(z_{s}-c\right)\right|<1 / 2$ for all $s=1, \ldots, n$.

(2.5) All $z_{1}, \ldots, z_{n}$ have the same real part and there is a subset $I \subset\{1, \ldots, n\}$ such that $\left|z_{r}-z_{s}\right|>\ell_{r}+\ell_{s}$ for all $r, s \in I$ and all $r, s \notin I$.

Condition (2.4) covers the most physically interesting and, perhaps, the most challenging case of the homogeneous $X X X$ model, $z_{1}=\ldots=z_{n}=0$.

\section{Algebraic Bethe ansatz}

The algebraic Bethe ansatz gives the following recipe to obtain eigenvectors and eigenvalues of the transfer-matrix $\mathcal{T}(u)$ on the spaces $\mathcal{H}_{k}$. Consider the reference state

$$
|\emptyset\rangle=w_{\ell_{1}} \otimes \ldots \otimes w_{\ell_{n}} \in \mathcal{H}_{0} .
$$

It is an eigenvector of the entries $A(u)$ and $D(u)$, and is annihilated by the entry $C(u)$ :

$$
A(u)|\emptyset\rangle=a(u)|\emptyset\rangle, \quad D(u)|\emptyset\rangle=d(u)|\emptyset\rangle, \quad C(u)|\emptyset\rangle=0,
$$

where

$$
a(u)=\prod_{s=1}^{n}\left(u-z_{s}+i \ell_{s}\right), \quad d(u)=\prod_{s=1}^{n}\left(u-z_{s}-i \ell_{s}\right) .
$$

Let $k$ be an integer such that $0 \leqslant k \leqslant \ell_{1}+\ldots+\ell_{n}$. Set

$$
\left|t_{1}, \ldots, t_{k}\right\rangle=B\left(t_{1}\right) \ldots B\left(t_{k}\right)|\emptyset\rangle .
$$

It is known that the vector $\left|t_{1}, \ldots, t_{k}\right\rangle$ is a symmetric function of $t_{1}, \ldots, t_{k}$.

The Bethe ansatz equations is the following system of algebraic equations on $t_{1}, \ldots, t_{k}$,

$$
a\left(t_{s}\right) \prod_{\substack{r=1 \\ r \neq s}}^{k}\left(t_{s}-t_{r}-i\right)=d\left(t_{s}\right) \prod_{\substack{r=1 \\ r \neq s}}^{k}\left(t_{s}-t_{r}+i\right), \quad s=1, \ldots, k .
$$

Given a solution of the Bethe ansatz equations, the algebraic Bethe ansatz suggests a presumable eigenvector of the transfer-matrix.

Theorem 3.1. Let $t_{1}, \ldots, t_{k}$ be a solution of system (3.3) such that

$$
t_{r} \neq t_{s}, \quad 1 \leqslant r<s \leqslant k .
$$

Then $\left|t_{1}, \ldots, t_{k}\right\rangle \in \mathcal{H}_{k}$ and

where

$$
\mathcal{T}(u)\left|t_{1}, \ldots, t_{k}\right\rangle=E\left(u ; t_{1}, \ldots, t_{k}\right)\left|t_{1}, \ldots, t_{k}\right\rangle,
$$

$$
E\left(u ; t_{1}, \ldots, t_{k}\right)=a(u) \prod_{s=1}^{k} \frac{u-t_{s}-i}{u-t_{s}}+d(u) \prod_{s=1}^{k} \frac{u-t_{s}+i}{u-t_{s}} .
$$


For a solution $t_{1}, \ldots, t_{k}$ of the Bethe ansatz equations, the vector $\left|t_{1}, \ldots, t_{k}\right\rangle$ is called the Bethe vector. The question whether the construction of Theorem 3.1 produces all eigenvectors of the transfer-matrix $\mathcal{T}(u)$ in $\mathcal{H}_{k}$ is called the question of completeness of the Bethe ansatz. A part of discussion of the completeness is counting solutions of the Bethe ansatz equations. Since both the vector $\left|t_{1}, \ldots, t_{k}\right\rangle$ and $E\left(u, t_{1}, \ldots, t_{k}\right)$ are symmetric functions of $t_{1}, \ldots, t_{k}$, this counting should not distinguish solutions of the Bethe ansatz equations obtained from each other by permutation of coordinates.

Introduce a polynomial $p(u)$ with the roots $t_{1}, \ldots, t_{k}$ :

$$
p(u)=\prod_{s=1}^{k}\left(u-t_{s}\right),
$$

We will always assume that $p(u)$ is related to $t_{1}, \ldots, t_{k}$ via $(3.5)$.

It turns out that assumption (3.4) that the roots of $p(u)$ are distinct can be eliminated.

Theorem 3.2. Assume that the ratio

$$
E(u ; p)=\frac{a(u) p(u-i)+d(u) p(u+i)}{p(u)} \quad \text { is a polynomial. }
$$

Then $\left|t_{1}, \ldots, t_{k}\right\rangle \in \mathcal{H}_{k}$ and

$$
\mathcal{T}(u)\left|t_{1}, \ldots, t_{k}\right\rangle=E(u ; p)\left|t_{1}, \ldots, t_{k}\right\rangle .
$$

The proof of this statement is similar to that of Theorem 3.1 and relies upon the following well known formulae that can be proved by induction on $k$ :

$$
\begin{aligned}
\mathcal{T}(u)\left|t_{1}, \ldots, t_{k}\right\rangle & =\frac{a(u) p(u-i)+d(u) p(u+i)}{p(u)}\left|t_{1}, \ldots, t_{k}\right\rangle+ \\
& +i \sum_{s=1}^{k} \frac{a\left(t_{s}\right) p\left(t_{s}-i\right)+d\left(t_{s}\right) p\left(t_{s}+i\right)}{\left(u-t_{s}\right) p^{\prime}\left(t_{s}\right)}\left|u, t_{1}, \ldots, t_{s-1}, t_{s+1}, \ldots, t_{n}\right\rangle, \\
S^{+}\left|t_{1}, \ldots, t_{k}\right\rangle & =i \sum_{s=1}^{k} \frac{a\left(t_{s}\right) p\left(t_{s}-i\right)+d\left(t_{s}\right) p\left(t_{s}+i\right)}{\left(u-t_{s}\right) p^{\prime}\left(t_{s}\right)}\left|t_{1}, \ldots, t_{s-1}, t_{s+1}, \ldots, t_{n}\right\rangle .
\end{aligned}
$$

However, instead of canceling the "unwanted terms" individually, all those terms in each formula are summed up, and the obtained expressions are simplified using identities for rational functions.

Condition (3.6) gives a system of algebraic equations on the coefficients of the polynomial $p(u)$ explicitly written as follows. Let $y(u ; p)$ be the remainder of division of the polynomial $a(u) p(u-i)+d(u) p(u+i)$ by $p(u)$, and consider its coefficients: $y(u ; p)=\sum_{s=1}^{k} y_{s}(p) u^{k-s}$. Then the system of equations defined by (3.6) is

$$
y_{s}(u ; p)=0, \quad s=1, \ldots, k .
$$

Condition (3.6), hence system (3.8) clearly implies equations (3.3), while under assumption (3.4), equations (3.3) and (3.8) are equivalent.

Notice that the eigenvalue $E(u, p)$ in formula (3.7) uniquely determines the polynomial $p(u)$ via relation (3.6), see Proposition 3.3 below. This observation implies that nonzero 
Bethe vectors $\left|t_{1}, \ldots, t_{k}\right\rangle$ are linearly independent as eigenvectors of the transfer-matrix $\mathcal{T}(u)$ with different eigenvalues.

Proposition 3.3. Let $f(u)$ be a monic polynomial such that $\operatorname{deg} f \leqslant \ell_{1}+\ldots+\ell_{n}$ and

$$
a(u) f(u-i)+d(u) f(u+i)=E(u ; p) f(u) .
$$

Then $f(u)=p(u)$.

Proof. By definition of $E(u, p)$, the polynomial $p(u)$ solves equation (3.9). And if $f(u)$ is a polynomial solution of $(3.9)$ not proportional to $p(u)$, then the polynomial

$$
Q(u)=p\left(u+\frac{i}{2}\right) f\left(u-\frac{i}{2}\right)-p\left(u-\frac{i}{2}\right) f\left(u+\frac{i}{2}\right)
$$

is nonzero and satisfy $a(u) Q\left(u-\frac{i}{2}\right)=d(u) Q\left(u+\frac{i}{2}\right)$. Taking the subleading coefficient in $u$ in this equality yields $\operatorname{deg} Q=2\left(\ell_{1}+\ldots+\ell_{n}\right)$. Hence,

$$
\operatorname{deg} f=\operatorname{deg} Q-\operatorname{deg} p+1 \geqslant \ell_{1}+\ldots+\ell_{n}+1
$$

since $\operatorname{deg} p \leqslant \ell_{1}+\ldots+\ell_{n}$.

One more requirement usually imposed on solutions of the Bethe ansatz equations is that

$$
\begin{array}{ll}
t_{r} \neq t_{s}+i, & r, s=1, \ldots, k, \\
a\left(t_{s}\right) \neq 0, & s=1, \ldots, k, \\
d\left(t_{s}\right) \neq 0, & s=1, \ldots, k .
\end{array}
$$

In other words, it is assumed that the polynomial $p(u)$ is coprime with each of the polynomials $p(u-i), a(u)$, and $d(u)$. It is easy to show that if $p(u)$ obeys (3.6), then each line in (3.10) implies the others, and there exist $r, s=1, \ldots, n$, and $m=0, \ldots, k-1$, such that $m=\ell_{r}+\ell_{s}+i\left(z_{r}-z_{s}\right)$ and $p\left(z_{r}-i \ell_{r}+i l\right)=0$ for any $l=0, \ldots, m$. However, under condition (2.3), one has

$$
\left|z_{r}-i \ell_{r}, z_{r}-i \ell_{r}+i, \ldots, z_{s}+i \ell_{s}, \tilde{t}_{m+2}, \ldots, \tilde{t}_{k}\right\rangle=0
$$

for any $\tilde{t}_{m+2}, \ldots, \tilde{t}_{k}$. Thus if assumption (3.10) fails, the Bethe vector $\left|t_{1}, \ldots, t_{k}\right\rangle$ vanishes and equality (3.7) becomes trivial.

Solutions of the Bethe ansatz equations satisfying conditions (3.10) are called admissible. It turns out that for an admissible solution of the Bethe ansatz equations, Theorem 3.2 does produce an eigenvector of the transfer-matrix.

Theorem 3.4. Let $p(u)$ obey conditions (3.6) and (3.10). Then $\left|t_{1}, \ldots, t_{k}\right\rangle \neq 0$.

If $p(u)$ is a nondegenerate solution of system (3.8), then the result follows from Theorem 3.6 below, which is a minor generalization of the Gaudin-Korepin determinant formula (3.11) for the norm of a Bethe vector. For degenerate solutions of system (3.8), the result of Theorem 3.4 is new, and its proof is similar to the proof of the analogous statement for the Gaudin model given in [MTV11].

Define a function $N\left(t_{1}, \ldots, t_{k}\right)$ by the rule

$$
C\left(t_{1}\right) \ldots C\left(t_{k}\right)\left|t_{1}, \ldots, t_{k}\right\rangle=N\left(t_{1}, \ldots, t_{k}\right)|\emptyset\rangle .
$$


This function is usually called the norm of vector $\left|t_{1}, \ldots, t_{k}\right\rangle$. The Gaudin-Korepin formula (3.11) shows that the norm of a Bethe vector is essentially given by the Jacobian of the Bethe ansatz equations.

Theorem $3.5([\mathrm{Ko}])$. Let $t_{1}, \ldots, t_{k}$ be a solution of system (3.3) such that $t_{r} \neq t_{s}$ for all $1 \leqslant r<s \leqslant k$. Then

$$
\begin{aligned}
N\left(t_{1}, \ldots, t_{k}\right) & \left.=(-i)^{k^{2}} \prod_{s=1}^{k} d\left(t_{s}\right) \prod_{r=1}^{k} \prod_{\substack{1 \leqslant r<s \leqslant k \\
\left(t_{r}-t_{s}\right)^{2}}} \frac{1}{\partial t_{s}}\left(a\left(t_{s}\right) \prod_{\substack{r=1 \\
r \neq s}}^{k}\left(t_{s}-t_{r}-i\right)-d\left(t_{s}\right) \prod_{\substack{r=1 \\
r \neq s}}^{k}\left(t_{s}-t_{r}+i\right)\right)\right]_{r, s=1}^{k} .
\end{aligned}
$$

Alternative proofs of formula (3.11) are given in [S], [TV].

Using the Vandermonde determinant, the Jacobian of system (3.3) in formula (3.11) can be converted into the Jacobian of system (3.8), see formula (3.12). This allows us to eliminate assumption (3.4) in Theorem 3.5.

Theorem 3.6. Let $p(u)$ be a solution of system (3.8). Then

$$
N\left(t_{1}, \ldots, t_{k}\right)=(-1)^{k} \prod_{s=1}^{k} d\left(t_{s}\right) \operatorname{det}\left(\frac{\partial y_{r}}{\partial p_{s}}\right)_{r, s=1}^{k}
$$

where $p_{1}, \ldots, p_{k}$ are the coefficients of $p(u): p(u)=u^{k}+\sum_{s=1}^{k} p_{s} u^{k-s}$.

Formula (3.12) follows from (3.11) by the deformation arguments using Proposition 3.7 below. Also, it is plausible that the proof of formula (3.11) given in [S] can be modified to work without assumption (3.4).

The next proposition established in [MTV5] is an important technically tool.

Proposition 3.7. For any $z_{1}, \ldots, z_{n}$, system (3.8) has finitely many solutions, and their number counted with multiplicities does not depend on $z_{1}, \ldots, z_{n}$. For generic $z_{1}, \ldots, z_{n}$, all solutions of system (3.8) are nondegenerate and satisfy conditions (3.4) and (3.10).

All statements in this section, except the assertion of Theorem 3.4 for degenerate solutions of the Bethe ansatz equations, are obtained by a "direct" analysis of the algebraic Bethe ansatz formulae. These statements show that the Bethe ansatz equations (3.8) can have at most $\operatorname{dim} \mathcal{H}_{k}$ admissible solutions. And if system (3.8) does have $\operatorname{dim} \mathcal{H}_{k}$ nondegenerate admissible solutions, then the corresponding Bethe vectors form a basis of $\mathcal{H}_{k}$, that is, the Bethe ansatz is complete. Moreover, the restriction of the transfer-matrix $\mathcal{T}(u)$ on $\mathcal{H}_{k}$ in this case is diagonalizable and has simple spectrum.

It is widely believed that the Bethe ansatz is complete for generic $z_{1}, \ldots, z_{n}$. It is indeed true, but the proof is not easy at all. Theorem 3.8 follows from the results of [MTV5], though the details are not straightforward.

Theorem 3.8. Let $z_{1}, \ldots, z_{n}$ be generic. Then for any integer $k$ such that $0 \leqslant k \leqslant$ $\ell_{1}+\ldots+\ell_{n}$, system (3.3) has exactly $\operatorname{dim} \mathcal{H}_{k}$ nondegenerate admissible solutions and the corresponding Bethe vectors form a basis of $\mathcal{H}_{k}$. Moreover, the spectrum of the transfermatrix $\mathcal{T}(u)$ is simple modulo natural degeneration. 
However, for particular $z_{1}, \ldots, z_{n}$, it can happen that the number of admissible solution of the Bethe ansatz equations is strictly less than required for completeness of the Bethe ansatz. The simplest example is given by the homogeneous spin- $1 / 2$ model with $n=4$, and goes back to $[\mathrm{AV}]$.

Example. Let $\ell_{s}=1 / 2, z_{s}=0, s=1, \ldots, 4$, and $k=2$. Then $\operatorname{dim} \mathcal{H}_{2}=2$, but there is only one quadratic polynomial $p(u)$ satisfying conditions (3.6) and (3.10):

$$
p(u)=u^{2}-\frac{1}{12}, \quad E(u)=2 u^{4}+3 u^{2}+\frac{13}{8} .
$$

It follows from Theorem 5.1 below that for the homogeneous spin-1/2 model with even $n$, the number of admissible solution of the Bethe ansatz equations for any $k \geqslant 2$ is strictly less than $\operatorname{dim} \mathcal{H}_{k}$.

\section{PAIRS OF POLYNOMials}

To deal with the question of completeness of the Bethe ansatz for arbitrary $z_{1}, \ldots, z_{n}$, one has to take into account solutions of the Bethe ansatz equations that do not satisfy additional conditions (3.10). And there are two natural questions coming out: to figure out selection rules picking up the solutions that give rise to eigenvalues of the transfer-matrix, and to show that all eigenvalues can be eventually obtained this way. The key observation to answer the first question is described in Theorem 4.1. The second question is worked out in Theorem 4.2 .

Define

$$
W(u)=\prod_{s=1}^{n} \prod_{r=1}^{2 \ell_{s}}\left(u-z_{s}+i\left(\ell_{s}-r+\frac{1}{2}\right)\right),
$$

and for $m=1,2, \ldots$,

$$
W_{m}(u)=\prod_{\substack{s=1 \\ 2 \ell_{s}+1 \geqslant m}}^{n} \prod_{r=0}^{2 \ell_{s}-m}\left(u-z_{s}+i\left(\ell_{s}-r-\frac{m}{2}\right)\right) .
$$

Clearly, $W_{1}(u)=W(u)$, and $W_{m}(u)=1$ if $m>2 \max \left\{\ell_{1}, \ldots, \ell_{n}\right\}$. Notice that

$$
a(u)=\frac{W\left(u+\frac{i}{2}\right)}{W_{2}(u)}, \quad d(u)=\frac{W\left(u-\frac{i}{2}\right)}{W_{2}(u)} .
$$

Theorem 4.1. Let the polynomial $p(u)$ satisfy conditions (3.6) and (3.10). Then there exists a polynomial $q(u)$ such that

$$
p\left(u+\frac{i}{2}\right) q\left(u-\frac{i}{2}\right)-p\left(u-\frac{i}{2}\right) q\left(u+\frac{i}{2}\right)=W(u)
$$

and

for any $m=2,3, \ldots$, the polynomial

$$
Q_{m}(u)=p\left(u+m \frac{i}{2}\right) q\left(u-m \frac{i}{2}\right)-p\left(u-m \frac{i}{2}\right) q\left(u+m \frac{i}{2}\right)
$$

is divisible by $W_{m}(u)$. (Recall that $W_{m}(u)=1$ if $m>2 \max \left\{\ell_{1}, \ldots, \ell_{n}\right\}$.) 
The eigenvalue $E(u, p)$ in Theorem 3.2 is expressed as

$$
E(u, p)=\frac{p(u+i) q(u-i)-p(u-i) q(u+i)}{W_{2}(u)} .
$$

The statement of Theorem 4.1 under assumption (3.4) goes back to [PS]. It was independently obtained in [MV2] in more general form. The proof of Theorem 4.1 avoiding (3.4) is similar to that of [LT, Proposition 4.1]. The part (4.5) for $m \geqslant 3$ is new. It follows by induction from the equality $Q_{1}(u)=W(u)$, see (4.4), and the recurrence relation

$$
Q_{m}(u) p\left(u+(m-2) \frac{i}{2}\right)=Q_{m-1}\left(u-\frac{i}{2}\right) p\left(u+m \frac{i}{2}\right)+Q_{1}\left(u+(m-1) \frac{i}{2}\right) p\left(u-m \frac{i}{2}\right)
$$

The next statement shows that under conditions (4.4), (4.5), formula (4.6) produce all eigenvalues of the transfer-matrix. The statement follows from the results of [MTV2], see Corollaries 7.4 and 7.5 therein.

Theorem 4.2. Let a polynomial $E(u)$ be an eigenvalue of the restriction of $\mathcal{T}(u)$ on $\mathcal{H}_{k}$. Then there are a unique monic polynomial $p(u)$ of degree $k$ and a polynomial $q(u)$ such that they satisfy conditions (4.4) and

$$
E(u)=\frac{p(u+i) q(u-i)-p(u-i) q(u+i)}{W_{2}(u)},
$$

cf. (4.6). Furthermore, the polynomials $p(u), q(u)$ satisfy condition (4.5).

Lemma 4.3. For a polynomial $E(u)$, the difference equation

$$
a(u) f(u-i)+d(u) f(u+i)=E(u) f(u)
$$

has two linearly independent polynomial solutions if and only if there are polynomials $p(u)$, $q(u)$ satisfying conditions (4.4) and (4.8). Moreover, if equation (4.9) has a polynomial solution that obey condition (3.10), then the polynomials $p(u), q(u)$ satisfy condition (4.5).

Proof. Clearly, if polynomials $p(u), q(u)$ satisfy conditions (4.4), (4.8), then they are linearly independent solutions of equation (4.9).

On the other hand, if $p(u), q(u)$ are linearly independent solutions of equations (4.9), then the polynomial

$$
Q_{1}(u)=p\left(u+\frac{i}{2}\right) q\left(u-\frac{i}{2}\right)-p\left(u-\frac{i}{2}\right) q\left(u+\frac{i}{2}\right)
$$

is nonzero and satisfy

$$
\frac{Q_{1}\left(u+\frac{i}{2}\right)}{Q_{1}\left(u-\frac{i}{2}\right)}=\frac{a(u)}{d(u)}=\frac{W\left(u+\frac{i}{2}\right)}{W\left(u-\frac{i}{2}\right)},
$$

see (4.3). Normalizing $p(u), q(u)$ such that $Q_{1}(u)$ is monic yields equality (4.4), while formula (4.6) follows from (4.9) with $f(u)=p(u)$.

To prove the second sentence of Lemma 4.3, notice that the polynomials $Q_{m}(u)$ do not depend up to proportionality on a choice of linearly independent solutions $p(u), q(u)$ of equation (4.9). Thus one may assume that $p(u)$ obeys (3.10), so the claim follows by induction from the equality $Q_{1}(u)=W(u)$ and relation (4.7). 


\section{COMPleteness FOR SPIN-1/2 MODEL}

In the next two sections we formulate the final results on completeness of the Bethe ansatz for the XXX model. We will describe eigenvectors and eigenvalues of the transfer-matrix in terms of pairs of polynomials $p(u), q(u)$ such that $\operatorname{deg} p<\operatorname{deg} q$ and $p(u)$ is a monic polynomial. We call such pairs ordered pairs of polynomials. By a minor abuse of notation, we will assume that in the ordered pair of polynomials $p(u), q(u)$, the polynomial $q(u)$ of the higher degree is determined only up to adding a multiple of the polynomial $p(u)$ of the lower degree.

Clearly, there is a bijection between ordered pairs of polynomials and two-dimensional subspaces of $\mathbb{C}[u]$ defined by assigning to an ordered pair of polynomials $p(u), q(u)$ the span of $p(u), q(u)$. Nevertheless, we prefer to talk about ordered pairs of polynomials rather than two-dimensional spaces of polynomials since the former has closer psychological association with the original Bethe ansatz equations.

Clearly, for an ordered pair of polynomials $p(u), q(u)$, any determinant $p\left(u_{1}\right) q\left(u_{2}\right)-$ $p\left(u_{2}\right) q\left(u_{1}\right)$ is not affected by the ambiguity in $q(u)$.

In this section we consider spin- $1 / 2$ models. That is, we take $\ell_{s}=1 / 2$ for all $s=1, \ldots$, $n$, so that $\mathcal{H}=\left(\mathbb{C}^{2}\right)^{\otimes n}$,

$$
a(u)=W\left(u+\frac{i}{2}\right), \quad d(u)=W\left(u-\frac{i}{2}\right), \quad W(u)=\prod_{s=1}^{n}\left(u-z_{s}\right) .
$$

We replace condition (2.3) by a weaker condition

$$
z_{s}-z_{r} \neq i, \quad 1 \leqslant r<s \leqslant n .
$$

This condition means that vectors $\left|t_{1}, \ldots, t_{k}\right\rangle$ with various $k=0, \ldots, n$, and $t_{1}, \ldots, t_{k}$ span the space $\mathcal{H}$, see $[\mathrm{T}]$.

Notice that for $\mathcal{H}=\left(\mathbb{C}^{2}\right)^{\otimes n}$, the subspace $\mathcal{H}_{k}$ is not trivial if and only if $k$ is an integer such that $0 \leqslant 2 k \leqslant n$. Farther on in this section, we will assume that $2 k \leqslant n$.

The next theorem is proved in [MTV5]. Similar result for the XXX-type integrable model associated with the Lie algebra $\mathfrak{g l}_{N}$, is obtained in [MTV9], see also [MTV7].

Theorem 5.1. Let $\ell_{s}=1 / 2$ for all $s=1, \ldots, n$, and $z_{1}, \ldots, z_{n}$ satisfy condition (5.2). A polynomial $E(u)$ is an eigenvalue of the restriction of $\mathcal{T}(u)$ on $\mathcal{H}_{k}$ if and only if there exists an ordered pair of polynomials $p(u), q(u)$ such that $\operatorname{deg} p=k$,

$$
\begin{gathered}
p\left(u+\frac{i}{2}\right) q\left(u-\frac{i}{2}\right)-p\left(u-\frac{i}{2}\right) q\left(u+\frac{i}{2}\right)=W(u), \\
p(u+i) q(u-i)-p(u-i) q(u+i)=E(u) .
\end{gathered}
$$

Moreover, the corresponding eigenvector is unique up to proportionality. In particular, if the restriction of $\mathcal{T}(u)$ on $\mathcal{H}_{k}$ is diagonalizable, then there exist exactly $\operatorname{dim} \mathcal{H}_{k}$ ordered pairs of polynomials $p(u), q(u)$ such that $\operatorname{deg} p=k$ and equality (5.3) holds.

Applying Lemma 4.3 gives another version of Theorem 5.1.

Theorem 5.2. Let $\ell_{s}=1 / 2$ for all $s=1, \ldots, n$, and $z_{1}, \ldots, z_{n}$ satisfy condition (5.2). A polynomial $E(u)$ is an eigenvalue of the restriction of $\mathcal{T}(u)$ on $\mathcal{H}_{k}$ if and only if the 
difference equation

$$
a(u) f(u-i)+d(u) f(u+i)=E(u) f(u)
$$

has polynomial solutions $p(u), q(u)$ such that $\operatorname{deg} p=k<\operatorname{deg} q$. Moreover the corresponding eigenvector is unique up to proportionality. In particular, if the restriction of $\mathcal{T}(u)$ on $\mathcal{H}_{k}$ is diagonalizable, then there exist exactly $\operatorname{dim} \mathcal{H}_{k}$ polynomials E(u) such that equation (5.5) has polynomial solutions $p(u), q(u)$ with $\operatorname{deg} p=k<\operatorname{deg} q$.

Suppose there exists an ordered pair of polynomials $p(u), q(u)$ such that $\operatorname{deg} p=k$, equality (5.3) holds, and the roots of $p(u)$ do not satisfy condition (3.10). Then by Theorems 5.1, 4.1, and 4.2, the number of admissible solutions of system (3.8) in this case is strictly less than the dimension of the space $\mathcal{H}_{k}$. The simplest example is the same as at the end of Section 3.

Example. Let $n=4, \ell_{s}=1 / 2, z_{s}=0, s=1, \ldots, 4$, and $k=2$. Then $\operatorname{dim} \mathcal{H}_{2}=2$, and there are two ordered pairs of polynomials $p(u), q(u)$ such that $\operatorname{deg} p=2$ and equality (5.3) holds: $p(u)=u^{2}+1 / 4, q(u)=i u\left(u^{2}+5 / 4\right)$, and $p(u)=u^{2}-1 / 12, q(u)=i u\left(u^{2}+1 / 4\right)$. However, only for the second pair, the roots of $p(u)$ satisfy condition (3.10) and give an admissible solution of system (3.8).

This example can be generalized. One can show that for the homogeneous spin-1/2 model, $z_{s}=0, s=1, \ldots, n$, with even $n$, for any $k \geqslant 2$, there exist ordered pairs of polynomials $p(u), q(u)$ such that $\operatorname{deg} p=k$, equality (5.3) holds, and $p(i / 2)=p(-i / 2)=0$, so the roots of $p(u)$ do not satisfy condition (3.10).

\section{Completeness FOR Higher SPIN MODEL}

In this section we return to the general case. Recall formulae $(4.1)-(4.3)$ :

$$
\begin{gathered}
W(u)=\prod_{s=1}^{n} \prod_{r=1}^{2 \ell_{s}}\left(u-z_{s}+i\left(\ell_{s}-r+\frac{1}{2}\right)\right), \\
W_{m}(u)=\prod_{\substack{s=1 \\
2 \ell_{s}+1 \geqslant m}}^{n} \prod_{r=0}^{2 \ell_{s}-m}\left(u-z_{s}+i\left(\ell_{s}-r-\frac{m}{2}\right)\right) .
\end{gathered}
$$

The next theorem is the main result of the paper.

Theorem 6.1. Let $z_{1}, \ldots, z_{n}$ satisfy condition (2.3). A polynomial $E(u)$ is an eigenvalue of the restriction of $\mathcal{T}(u)$ on $\mathcal{H}_{k}$ if and only if there exists an ordered pair of polynomials $p(u), q(u)$ such that $\operatorname{deg} p=k$,

$$
p\left(u+\frac{i}{2}\right) q\left(u-\frac{i}{2}\right)-p\left(u-\frac{i}{2}\right) q\left(u+\frac{i}{2}\right)=W(u),
$$

for any $m=2,3, \ldots$, the polynomial

$$
Q_{m}(u)=p\left(u+m \frac{i}{2}\right) q\left(u-m \frac{i}{2}\right)-p\left(u-m \frac{i}{2}\right) q\left(u+m \frac{i}{2}\right)
$$

is divisible by $W_{m}(u)$, and

$$
\frac{p(u+i) q(u-i)-p(u-i) q(u+i)}{W_{2}(u)}=E(u),
$$


(Recall that $W_{m}(u)=1$ if $\left.m>2 \max \left\{\ell_{1}, \ldots, \ell_{n}\right\}.\right)$ Moreover, the corresponding eigenvector is unique up to proportionality. In particular, if the restriction of $\mathcal{T}(u)$ on $\mathcal{H}_{k}$ is diagonalizable, then there exist exactly $\operatorname{dim} \mathcal{H}_{k}$ ordered pairs of polynomials $p(u), q(u)$ such that $\operatorname{deg} p=k$, equality (5.3) holds, and for any $m=2,3, \ldots$, the polynomial $Q_{m}(u)$ is divisible by $W_{m}(u)$.

Theorem 6.1 is similar to Theorem 5.1. However, there is no counterpart of Theorem 5.2 for the higher spin case. If the difference equation

$$
a(u) f(u-i)+d(u) f(u+i)=E(u) f(u)
$$

has two linearly independent polynomial solutions $p(u), q(u)$, then they should obey relations (6.1) and (6.3). But the next illuminating example shows that the divisibility of $Q_{2}(u)$ by $W_{2}(u)$ given by $(6.3)$ need not imply the divisibility of $Q_{m}(u)$ by $W_{m}(u)$ for $m \geqslant 3$.

Example. Let $n=4, \ell_{1}=\ell_{2}=1 / 2, \ell_{3}=\ell_{4}=3 / 2, z_{s}=0, s=1, \ldots, 4$, and $k=4$, so that

$$
W(u)=u^{4}\left(u^{2}+1\right)^{2}, \quad W_{2}(u)=\left(u^{2}+\frac{1}{4}\right)^{2}, \quad W_{3}(u)=u^{2} .
$$

Then $\operatorname{dim} \mathcal{H}_{4}=2$, but there are three ordered pairs of polynomials $p(u), q(u)$ such that $\operatorname{deg} p=4$, equality (6.1) holds, and the polynomial $Q_{2}(u)$ is divisible by $W_{2}(u)$ :

$$
\begin{array}{ll}
p(u)=u^{4}+\frac{7}{6} u^{2}-\frac{3}{80}, & q(u)=i u\left(u^{2}+\frac{1}{4}\right)\left(u^{2}+\frac{9}{4}\right), \\
p(u)=\left(u^{2}+\frac{1}{4}\right)\left(u^{2}+\frac{9}{4}\right), & q(u)=i u\left(u^{4}+\frac{13}{2} u^{2}+\frac{89}{16}\right), \\
p(u)=\left(u^{2}+\frac{1}{4}\right)^{2}, & q(u)=i u\left(u^{2}+\frac{1}{4}\right)^{2} .
\end{array}
$$

Among these three pairs, only for one of them, (6.4), the roots of $p(u)$ satisfy condition (3.10), and exactly for two of them, (6.4) and (6.5), the polynomial $Q_{3}(u)$ is divisible by $W_{3}(u)$. Therefore, the eigenvalues of the restriction of $\mathcal{T}(u)$ on $\mathcal{H}_{4}$ are given by the polynomials $2 u^{4}+9 u^{2}-15 / 8$ and $2 u^{4}+9 u^{2}+49 / 8$ corresponding by formula (6.3) to pairs (6.4), (6.5), respectively, while the polynomial $2 u^{4}+9 u^{2}+81 / 8$ corresponding to pair (6.6) is not an eigenvalue of $\mathcal{T}(u)$.

Nevertheless, Theorem 5.2 survives almost literally for higher spin models satisfying the following condition:

Let $X_{s}=\left\{z_{s}-i \ell_{s}, z_{s}-i\left(\ell_{s}-1\right), \ldots, z_{s}+i \ell_{s}\right\}, s=1, \ldots, n$, Then for any $r, s=1$, $\ldots, n$, the sets $X_{r}$ and $X_{s}$ either do not intersect or coincide.

cf. (2.3). In particular, condition (6.7) holds for homogeneous models. Notice that if $X_{r}=X_{s}$ in (6.7), then $z_{r}=z_{s}$ and $\ell_{r}=\ell_{s}$.

Theorem 6.2. Let $z_{1}, \ldots, z_{n}$ satisfy condition (6.7). A polynomial $E(u)$ is an eigenvalue of the restriction of $\mathcal{T}(u)$ on $\mathcal{H}_{k}$ if and only if the difference equation

$$
a(u) f(u-i)+d(u) f(u+i)=E(u) f(u)
$$

has polynomial solutions $p(u), q(u)$ such that $\operatorname{deg} p=k<\operatorname{deg} q$. Moreover the corresponding eigenvector is unique up to proportionality. In particular, if the restriction of $\mathcal{T}(u)$ on $\mathcal{H}_{k}$ is diagonalizable, then there exist exactly $\operatorname{dim} \mathcal{H}_{k}$ polynomials $E(u)$ such that equation (5.5) has polynomial solutions $p(u), q(u)$ with $\operatorname{deg} p=k<\operatorname{deg} q$. 
Theorem 6.2 follows from Theorem 6.1 by Lemma 4.3 and the next statement.

Lemma 6.3. Let $z_{1}, \ldots, z_{n}$ satisfy condition (6.7). Assume that the polynomials $p(u), q(u)$ satisfy condition (6.1), and the polynomial $Q_{2}(u)$ is divisible by $W_{2}(u)$. Then the polynomial $Q_{m}(u)$ is divisible by $W_{m}(u)$ for all $m \geqslant 3$.

Proof. The statement follows by induction on $m$ from the next Plücker relation

$$
\begin{aligned}
& Q_{m}(u) Q_{1}\left(u+(m-3) \frac{i}{2}\right)= \\
& =Q_{m-1}\left(u-\frac{i}{2}\right) Q_{2}\left(u+(m-2) \frac{i}{2}\right)-Q_{m-2}(u-i) Q_{1}\left(u+(m-1) \frac{i}{2}\right) .
\end{aligned}
$$

\section{REFERENCES}

[AV] L.V. Avdeev, A. A. Vladimirov, Exceptional solutions of the Bethe ansatz equations, Teor. Matem. Fiz. 69 (1986), no. 2, 163-174 (in Russian); Theor. Math. Phys. 69 (1987), no. 2, 1071-1079.

[B1] R. Baxter, Exactly solved models in statistical mechanics, Academic Press, Inc., London, 1982.

[B2] R. Baxter, Completeness of the Bethe ansatz for the six- and eight-vertex models, J. Stat. Phys. 108 (2002), no. 1-2, 1-48.

[Be] H. Bethe, Zur Theorie der Metalle: I. Eigenwerte und Eigenfunktionen der linearen Atomkette, Z. Phys. 71 (1931), 205-226.

[FFR] B. Feigin, E. Frenkel, L. Rybnikov, Opers with irregular singularity and spectra of the shift of argument subalgebra, Duke Math. J. 155 (2010), no. 2, 337-363.

[FT] L. D. Faddeev, L. A. Takhtajan, The quantum method for the inverse problem and the XYZ Heisenberg model, Uspekhi Matem. Nauk 34 (1979), no. 5, 13-63 (in Russian); Russian Math. Surveys 34 (1979), no. 5, 11-68.

[FT] L. D. Faddeev, L. A. Takhtajan, The spectrum and scattering of excitations in the onedimensional isotropic Heisenberg model, Zap. Nauch. Semin. LOMI 109 (1981), 134-178 (in Russian); J. Soviet Math. 24 (1984), 241-267.

[DG] P. R. Giri, T. Deguchi, Singular eigenstates in the even (odd) length Heisenberg spin chain, J. Phys. A 48 (2015), no. 17, 175207, 1-26.

T. Deguchi, P. R. Giri, Exact quantum numbers of collapsed and non-collapsed 2-string solutions in the Heisenberg spin chain, J. Phys. A 49 (2016), no. 17, 174001, 1-24.

[GN] A. Gainutdinov, R. Nepomechie, Algebraic Bethe ansatz for the quantum group invariant open $X X Z$ chain at roots of unity, Nucl. Phys. B 909, (2016), 796-839.

[HNS] W. Hao, R. Nepomechie, A. Sommes, Singular solutions, repeated roots and completeness for higher-spin chains, J.;Stat. Mech. (2014), no. 3, P03024, 1-20.

[Ki] A. N. Kirillov, Combinatorial identities and the completeness of states for Heisenberg magnet, Zap. Nauch. Semin. LOMI 131 (1983), 88-105 (in Russian); J. Soviet Math. 30 (1985), 2298-3310.

A. N. Kirillov, Completeness of the states for the generalized Heisenberg model, Zap. Nauch. Semin. LOMI 134 (1985), 169-189 (in Russian); J. Soviet Math. 36 (1987), $115-128$.

[KS] A. N. Kirillov, R. Sakamoto, Singular solutions to the Bethe ansatz equations and rigged configurations, J. Phys. A 47 (2014), no. 20, 205207, 1-20.

A. N. Kirillov, R. Sakamoto, Bethe's quantum numbers and rigged configurations, Nucl. Phys. B 905 (2016), 359-372. 
[Ko] V.E. Korepin, Calculation of norms of Bethe wave functions, Commun. Math. Phys. 86 (1982), no. 3, 391-418.

[KBI] V.E. Korepin, N. M. Bogoliubov, A. G. Izergin, Quantum inverse scattering method and correlation functions, Cambridge University Press, 1993.

[KR] P. P. Kulish, N. Yu. Reshetikhin, Quantum linear problem for the sine-Gordon equation and higher representations, Zap. Nauch. Sem. LOMI 101 (1981), 101-110 (in Russian); J. Soviet Math. 23 (1983), no. 4, 2435-2441.

[KRS] P.P. Kulish, N.Yu. Reshetikhin, E. K.Sklyanin, Yang-Baxter equations and representation theory I, Lett. Math. Phys. 5 (1981), no. 5, 393-403.

[LS] R.P. Langlands, Y.Saint-Aubin Combinatorial aspects of the Bethe equations, CRM Proc. Lect. Notes 11 (1997), 231-301.

[LT] J.R. Li, V. Tarasov, XXZ-type Bethe ansatz equations and quasi-polynomials, J. Phys.: Conf. Ser. 411 (2013), 012020, 1-20.

[MTV0] E. Mukhin, V. Tarasov, A.Varchenko, Schubert calculus and representations of the general linear group J. Amer. Math. Soc. 22 (2009), no. 4, 909-940.

[MTV2] E. Mukhin, V. Tarasov, A. Varchenko, Generating operator of XXX or Gaudin transfer matrices has quasi-exponential kernel, SIGMA 6 (2007), 060, 1-31.

[MTV5] E. Mukhin, V. Tarasov, A.Varchenko, Bethe algebra of homogeneous XXX Heisenberg model has simple spectrum, Commun. Math. Phys. 288 (2009), no. 1, 1-42.

[MTV7] E. Mukhin, V. Tarasov, A.Varchenko, Bethe subalgebras of the group algebra of the symmetric group, Transform. Groups 18 (2013), no. 3, 767-801.

[MTV9] E. Mukhin, V. Tarasov, A.Varchenko, Spaces of quasi-exponentials and representations of the Yangian $Y\left(\mathfrak{g l}_{N}\right)$, Transform. Groups 19 (2014), no. 3, 861-885.

[MTV10] E. Mukhin, V. Tarasov, A.Varchenko, On reality property of Wronski maps, Confluentes Math. 1 (2009), no. 2, 225-247.

E. Mukhin, V. Tarasov, A.Varchenko, Reality property of discrete Wronski map with imaginary step, Lett. Math. Phys. 100 (2012), no. 2, 151-160. 
[MTV11] E. Mukhin, V. Tarasov, A.Varchenko, Bethe algebra of the $\mathfrak{g l}_{N+1}$ Gaudin model and algebra of functions on the critical set of the master function, New trends in Quantum Integrable Systems (B. Feigin, M. Jimbo, M. Okado, eds.) 307-324, World Scientific, 2011.

[MV2] E. Mukhin and A.Varchenko, Solutions to the XXX type Bethe ansatz equations and flag varieties, Cent. Eur. J. Math. 1 (2003), no. 2, 238-271.

[PS] G.P.Pronko and Yu. G.Stroganov, Bethe equations "on the wrong side of equator", J. Phys. A 32 (1999), no. 12, 2333-2340.

[R] L. Rybnikov, A proof of the Gaudin Bethe ansatz conjecture, Preprint (2016), 1-15; arXiv:1608.04625.

[S] N. A. Slavnov, Calculation of scalar products of wave functions and form-factors in the framework of the algebraic Bethe ansatz, Teor. Matem. Fiz. 79 (1989), no. 2, 232-240 (in Russian); Theor. Math. Phys. 79 (1989), no. 2, 502-508.

N. A. Slavnov, The Algebraic Bethe ansatz and quantum integrable systems, Uspekhi Matem. Nauk 62 (2007), no. 4, 91-132 (in Russian); Russian Math. Surveys 62 (2007), no. 4, 727-766.

[T] V. Tarasov, Irreducible monodromy matrices for the R-matrix of the XXZ-model and lattice local quantum Hamiltonians, Teor. Matem. Fiz. 63 (1985), no, 2, 175-196 (in Russian); Theor. Math. Phys. 63 (1985), no, 2, 440-454.

[TV] V. Tarasov, A.Varchenko, Completeness of Bethe vectors and difference equations with regular singular points, Intern. Math. Res. Notices (1995), no. 13, 637-669.

Published in Ludwig Faddeev Memorial Volume: A Life in Mathematical Physics, (Mo-Lin Ge, Antti J. Niemi, Kok Khoo Phua and Leon A.Takhtajan, eds.), World Scientic, 2018, pp. 549-566. https://doi.org/10.1142/9789813233867_0026

Reprinted in Reviews in Math. Phys. 30 (2018), no. 8, 1840018, 1-18. http://dx.doi.org/10.1142/S0129055X18400184 